\title{
AUFSATZ
}

\section{Die Reform des Sozialstaats und das deutsche Parteiensystem: Abschied von den Volksparteien?*}

\author{
Armin Schäfer
}

Das Ergebnis der Bundestagswahl 2005 löste bei den politischen Akteuren Entsetzen aus. Nach einem kurzen Wahlkampf, in dem sich die Volksparteien heftig attackiert hatten, erzwang das doppelte Nein der Wähler - das Nein zur Politik der rot-grünen Bundesregierung und das Nein zur schwarz-gelben Alternative - die Zusammenarbeit in einer ungeliebten Großen Koalition. Betrachtet man die Entwicklung des deutschen Parteiensystems über einen längeren Zeitraum, überrascht das Ergebnis weniger. Seit dem Höhepunkt in den siebziger Jahren geht der Stimmenanteil zurück, den die Volksparteien auf sich vereinen. Wie in anderen westeuropäischen Ländern lässt sich auch in Deutschland eine Abkehr der Wähler von sozial- und christdemokratischen Parteien beobachten. ${ }^{1}$ Einschnitte ins soziale Netz haben einen Teil der Stammwähler der beiden deutschen „Sozialstaatsparteien" 2 verprellt. Die Reform des Sozialstaats, die häufig den Abbau sozialer Leistungen beinhaltet, wird in vielen Fällen von einer Mehrheit der Bevölkerung abgelehnt.

Die Kluft zwischen den Wählerpräferenzen und einer „Politik der Zumutungen“3 übt erheblichen Druck auf die Volksparteien aus. In der SPD sind die resultierenden Spannungen offensichtlich. So begründete Gerhard Schröder seine Entscheidung für Neuwahlen auch mit innerparteilichem Widerstand gegen die Agenda 2010. Aber auch in der CDU herrscht über den richtigen sozialpolitischen Kurs Streit zwischen dem christlich-sozialen, arbeitnehmernahen Flügel und stärker marktliberalen Positionen. Für beide Parteien besteht die Schwierigkeit darin, dass weder eine „Politik der ruhigen Hand“ noch eine energische Liberalisierungspolitik kurzfristig von den Wählern belohnt wird. Wie Oskar Niedermayer betont, reagierte die SPD mit der Agenda 2010 auf vorausgegangene Wahlniederlagen,

Dieser Aufsatz entstand während eines Gastaufenthalts am Hanse-Wissenschaftskolleg in Delmenhorst. Für hilfreiche Hinweise danke ich Holger Döring, Saskia Freye, Achim Goerres, Martin Höpner, Simone Leiber, Mark Lutter und Hendrik Zorn.

1 Vgl. Hans Keman / Kees van Kersbergen / Barbara Vis, Political Parties and New Social Risks: The Double Backlash Against Social Democracy and Christian Democracy, in: Klaus Armingeon I Giuliano Bonoli (Hrsg.), The Politics of Post-Industrial Welfare States. Adapting Post-war Social Policies to New Social Risks, London / New York 2006, S. 27 - 51; Fraser Duncan, A Decade of Christian Democratic Decline: The Dilemmas of the CDU, ÖVP and CDA in the 1990s, in: Government and Opposition, 41. Jg. (2006), S. $469-490$.

2 Manfred G. Schmidt, Wenn zwei Sozialstaatsparteien konkurrieren: Sozialpolitik in Deutschland, in: ders. / Reimut Zohlnhöfer (Hrsg.), Regieren in der Bundesrepublik Deutschland: Innen- und Außenpolitik seit 1949, Wiesbaden 2006, S. 137 - 157.

3 Elmar Wiesendahl, Parteien und die Politik der Zumutungen, in: APuZ, B 40 (2004), S. 19 - 24. 
bei denen sie für die schlechte wirtschaftliche Entwicklung und steigende Arbeitslosigkeit abgestraft worden war. ${ }^{4}$ Als sich Bundeskanzler Schröder schließlich für Reformen entschied, verbesserte dies - auch aufgrund einer schlecht begründeten und unausgewogenen Politik - die Lage der SPD nicht. ${ }^{5}$ Diese Erfahrungen lassen sich verallgemeinern. Angesichts des breiten Sozialstaatskonsenses der Bevölkerung stehen beide Sozialstaatsparteien vor der Herausforderung, Reformen gegen die eigene Wählerschaft durchzusetzen und so ihre Wiederwahl zu gefährden. ${ }^{6}$

In der Literatur wird zumeist angenommen, dass die Reform des Sozialstaats vor allem für die Sozialdemokratie politisch riskant ist. So sieht Herbert Kitschelt ${ }^{7}$ das Dilemma sozialdemokratischer Parteien darin, dass sie in Regierungsverantwortung zu wirtschaftsliberalen Reformen gezwungen sind, die sich negativ auf künftige Wahlergebnisse auswirken. Insbesondere linke und linkslibertäre Parteien können sich in dieser Situation als Verteidiger des Sozialstaats profilieren. Im Gegensatz dazu bleiben konservative Parteien, die ohnehin einer übertriebenen Sozialstaatsneigung unverdächtig sind, von negativen Reaktionen ihrer Wähler weitgehend verschont. Doch gilt diese Vermutung für christdemokratische Parteien nicht, da deren Geschichte eng mit dem Aufbau der kontinentaleuropäischen Sozialstaaten verknüpft ist. ${ }^{8}$ Im Folgenden soll daher argumentiert werden, dass die CDU als klassische Sozialstaatspartei - vor ähnlichen Herausforderungen wie die SPD steht. Mehr noch, unter der Bedingung eines nach links ausdifferenzierten Fünfparteiensystems befindet sich die SPD in einer strategisch günstigeren Position als die Union, weil sie mehr Koalitionsoptionen besitzt. Zudem weisen die Wähler eine ausgeprägte Sozialstaatsorientierung auf und versagen radikalen Reformen die Unterstützung.

Um dies zu zeigen, werden im zweiten Abschnitt Unterschiede und Gemeinsamkeiten zwischen dem ost- und dem westdeutschen Parteiensystem dargestellt. Dabei werden alle Landtags- und Bundestagswahlen seit 1990 untersucht. Während in Westdeutschland bürgerliche und Mitte-links-Parteien nahezu gleich stark sind, dominieren in Ostdeutschland Parteien links der Mitte. Vor diesem Hintergrund wird im dritten Abschnitt die Koalitionsarithmetik in Bund und Ländern nachgezeichnet. Durch die strukturelle linke Mehrheit in Ostdeutschland ergeben sich auch auf Bundesebene neue Koalitionsdynamiken. Nicht länger ist die FDP das für die Koalitionsbildung entscheidende Zünglein an der Waage, sondern die SPD. Ohne sie ist eine Koalitionsbildung kaum möglich. Gerade die Schwäche der beiden Volksparteien erhöht für die SPD die Wahrscheinlichkeit, in der Regierung

4 Vgl. Oskar Niedermayer, War die Agenda 2010 an allem Schuld? Die Regierungsparteien SPD und Bündnis 90/Die Grünen, in: Eckhard Jesse / Roland Sturm (Hrsg.), Bilanz der Bundestagswahl 2005. Voraussetzungen, Ergebnisse, Folgen, Wiesbaden 2006, S. 119 - 155, S. 121 - 130.

5 Joachim Raschke zieht folgende Bilanz der Agenda 2010: „Eine Reformpolitik, der die Begriffe fehlen, die dem gegnerischen Lager zugeordnet wird, von den eigenen Leuten an Podiumstischen, in Betrieben und auf Marktplätzen nicht erklärt werden kann, ist - ob richtig oder falsch - kommunikativ ein Desaster." Joachim Raschke, Rot-grüne Zwischenbilanz, in: APuZ, B 40 (2004), S. 25 - 31, S. 29.

6 Vgl. Reimut Zohlnhöfer, Die Wirtschaftspolitik der rot-grünen Koalition: Ende des Reformstaus?, in: ZPol, 14. Jg. (2004), S. 381 - 402, S. 395.

7 Herbert Kitschelt, European Social Democracy between Political Economy and Electoral Competition, in: ders. u.a. (Hrsg.), Continuity and Change in Contemporary Capitalism, Cambridge 1999, S. $317-345$.

8 Vgl. Kees van Kersbergen, Social Capitalism: A Study of Christian Democracy and the Welfare State, London 1995. 
vertreten zu sein. Der vierte Abschnitt untersucht die Einstellung der Bevölkerung zum Sozialstaat. Umfragen legen nahe, dass es keine Mehrheit für harte Einschnitte in die Systeme sozialer Sicherung gibt, wie sie etwa 2003 auf dem Leipziger Parteitag der CDU beschlossen wurden. Nicht nur von ostdeutschen Wählern, sondern auch im Westen wird ein umfassender Sozialstaat von einer Mehrheit befürwortet. Der letzte Abschnitt resümiert, was aus den empirischen Befunden für die Volksparteien folgt.

\section{Zwei deutsche Parteiensysteme?}

In zahlreichen Untersuchungen konnte gezeigt werden, dass sich die politischen Orientierungen von Ost- und Westdeutschen weiterhin unterscheiden. ${ }^{9}$ In der DDR sozialisierte Menschen stehen der Idee des Sozialismus häufiger positiv gegenüber und befürworten staatliche Eingriffe zur Korrektur von Marktergebnissen. ${ }^{10}$ Diese Einstellungsunterschiede, wie auch das Gefühl, zu den Wendeverlierern zu gehören, schlagen sich im Wahlverhalten, insbesondere im Abschneiden der Linkspartei/PDS nieder. ${ }^{11}$ In einer umfassenden Analyse der Landtags- und Bundestagswahlen zwischen 1990 und 2003 weisen Kai Arzheimer und Jürgen Falter $^{12}$ deutliche Unterschiede zwischen beiden Landesteilen nach. Dennoch befindet sich das deutsche Parteiensystem in keinem stationären Zustand. Dies zeigt sich daran, dass zwei Ergebnisse ihrer Analyse nur noch eingeschränkt gelten: Zum einen gewann die Linkspartei bei der Bundestagswahl 2005 erstmals auch im Westen in erheblichem Umfang Stimmen. Durch den Zusammenschluss mit der WASG haben sich die Chancen für eine Westausdehnung der PDS vergrößert. Bei der bremischen Bürgerschaftswahl im Mai 2007 zog die Linke zum ersten Mal in ein westdeutsches Landesparlament ein. Zum anderen lässt sich nicht länger aufrechterhalten, dass rechte Parteien im Westen erfolgreicher sind. In Brandenburg, Mecklenburg-Vorpommern und Sachsen haben Rechtsextreme den Einzug in die Landtage geschafft, wohingegen im Westen nur die DVU in der Bremer Bürgerschaft vertreten ist. Auch bei der Bundestagswahl 2005 schnitt die NPD mit 3,6 Prozent der gültigen Stimmen in Ostdeutschland wesentlich besser als in Westdeutschland ab (1,1 Prozent).

Im Folgenden wird die Entwicklung des deutschen Parteiensystems auf Grundlage der fünf Bundestagswahlen und der 69 Landtagswahlen seit 1990 untersucht. Tabelle 1 zeigt, dass sich die Wahlergebnisse vor allem von SPD und Linkspartei/PDS bei Landtagswahlen signifikant zwischen den Landesteilen unterscheiden. Während die PDS im Osten gut abschneidet, liegt der Schwerpunkt der SPD im Westen. Interessant ist das Ergebnis der in

9 Siehe die Beiträge in: Jürgen Falter / Oscar W. Gabriel / Hans Rattinger (Hrsg.), Wirklich ein Volk? Die politischen Orientierungen von Ost- und Westdeutschen im Vergleich, Opladen 2000.

10 Da die Sozialisation ein wichtiger Erklärungsfaktor ist, gibt es Anzeichen für eine Annäherung der Einstellungen jüngerer Alterskohorten, siehe: Kai Arzheimer / Markus Klein, Gesellschaftspolitische Wertorientierungen und Staatszielvorstellungen im Ost-West-Vergleich, in: Jürgen Falter I Oscar W. Gabriel / Hans Rattinger (Hrsg.), Wirklich ein Volk?, a.a.O., S. 363 - 402.

11 Vgl. Harald Schoen / Jürgen W. Falter, Die Linkspartei und ihre Wähler, in: APuZ, B 51/52 (2005), S. $33-40$.

12 Kai Arzheimer / Jürgen W. Falter, „Goodbye Lenin?“ Bundestags- und Landtagswahlen seit 1990: Eine Ost-West-Perspektive, in: Jürgen W. Falter / Oscar W. Gabriel / Bernhard Weßels (Hrsg.), Wahlen und Wähler: Analysen aus Anlass der Bundestagswahl 2002, Wiesbaden 2005, S. 244 -283 . 


\begin{tabular}{|c|c|c|c|c|c|c|}
\hline \multicolumn{7}{|c|}{$\begin{array}{l}\text { Tabelle 1: Regionale und zeitliche Trends der Wablergebnisse bei Landtagswablen } \\
\text { in der Bundesrepublik, } 1990 \text { bis } 2007\end{array}$} \\
\hline & SPD & $\begin{array}{l}\text { CDU / } \\
\text { CSU }\end{array}$ & $\begin{array}{l}\text { Links- } \\
\text { partei }\end{array}$ & $\begin{array}{l}\text { B'90 / } \\
\text { Grüne }\end{array}$ & FDP & Rechte \\
\hline $\begin{array}{l}\text { Region } \\
\text { (1= Ostdeutschland) }\end{array}$ & $\begin{array}{l}-10,6^{* * *} \\
(2,8)\end{array}$ & $\begin{array}{l}-2,6 \\
(2,9)\end{array}$ & $\begin{array}{l}11,6^{* * *} \\
(1,5)\end{array}$ & $\begin{array}{l}-1,4 \\
(0,9)\end{array}$ & $\begin{array}{l}-0,4 \\
(0,8)\end{array}$ & $\begin{array}{l}-2,4^{* *} \\
(0,7)\end{array}$ \\
\hline $\begin{array}{l}\text { Zeit } \\
\text { (Jahre seit 1990) }\end{array}$ & $\begin{array}{l}-0,6^{* * *} \\
(0,2)\end{array}$ & $\begin{array}{l}-0,3 \\
(0,2)\end{array}$ & $\begin{array}{c}0,1 \\
(0,1)\end{array}$ & $\begin{array}{c}0,0 \\
(0,1)\end{array}$ & $\begin{array}{l}-0,0 \\
(0,1)\end{array}$ & $\begin{array}{l}-0,1^{* *} \\
(0,0)\end{array}$ \\
\hline Zeit ${ }^{*}$ Region & $\begin{array}{c}0,2 \\
(0,3)\end{array}$ & $\begin{array}{l}-0,3 \\
(0,3)\end{array}$ & $\begin{array}{c}0,2 \\
(0,1)\end{array}$ & $\begin{array}{l}-0,1 \\
(0,1)\end{array}$ & $\begin{array}{l}-0,1 \\
(0,1)\end{array}$ & $\begin{array}{l}0,3^{* * *} \\
(0,1)\end{array}$ \\
\hline Konstante & $\begin{array}{c}30,8^{* * *} \\
(1,7)\end{array}$ & $\begin{array}{l}29,4^{* * *} \\
(1,8)\end{array}$ & $\begin{array}{l}-0,2 \\
(0,9)\end{array}$ & $\begin{array}{l}5,4^{* * *} \\
(0,6)\end{array}$ & $\begin{array}{l}3,9^{* * *} \\
(0,5)\end{array}$ & $\begin{array}{l}3,2^{* * *} \\
0,5)\end{array}$ \\
\hline korr. $\mathrm{R}^{2}$ & 0,39 & 0,23 & 0,78 & 0,29 & 0,03 & 0,13 \\
\hline \multicolumn{7}{|c|}{$\begin{array}{l}\mathrm{N}=74 \text { (= } 69+5 \text { Landtagswahlen; die Wahlergebnisse in Ost- und Westberlin werden getrennt betrach- } \\
\text { tet). Rechte = DVU, Republikaner, NPD. }{ }^{*} \mathrm{p}<0,05 ;{ }^{* *} \mathrm{p}<0,01 ;{ }^{* * *} \mathrm{p}<0,001 . \text { In Klammern: Standardfehler. } \\
\text { Das Regressionsmodell ist wie folgt zu lesen: Für die SPD wird ein Ergebnis von } 30,8 \text { Prozent erwartet } \\
\text { (Konstante). Dieses Ergebnis vermindert sich um } 10,6 \text { Prozentpunkte, wenn die Landtagswahl in einem } \\
\text { neuen Bundesland stattfindet, und um zusätzliche 0,6 Prozentpunkte mit jedem Jahr, das seit } 1990 \\
\text { vergangen ist. Der Produktterm aus Zeit und Region gibt an, ob eine regional spezifische Entwicklung } \\
\text { in Ostdeutschland vorliegt. Geschätzt werden die Wahlergebnisse in Prozent der Wahlberechtigten. } \\
\text { Quelle: Eigene Berechnung auf Grundlage der Daten des Bundeswahlleiters und www.election.de. }\end{array}$} \\
\hline
\end{tabular}

der Sammelkategorie „Rechte“ erfassten Parteien DVU, NPD und Republikaner. Aus den Koeffizienten geht hervor, dass diese Parteien Anfang der neunziger Jahre im Westen erfolgreicher als im Osten waren, dass sich dieser Trend in den letzten Jahren jedoch ins Gegenteil verkehrt hat. Liegt das Ergebnis für alle Wahlen in ostdeutschen Bundesländern unter dem in westdeutschen, weist der Interaktionsterm, der gezielt die Entwicklung in Ostdeutschland erfasst, einen positiven, signifikanten Wert auf. Für die anderen Parteien lässt sich kein klarer Trend erkennen. Die Liberalen konnten nach anfänglichen Erfolgen in Ostdeutschland erst in letzter Zeit dort wieder Zugewinne erzielen. Die Grünen sind 2007 nur im Sächsischen Landtag vertreten und im Kern eine westdeutsche Partei geblieben. ${ }^{13}$

Aus Tabelle 2 geht das Abschneiden der Parteien bei den fünf Bundestagswahlen seit 1990 hervor. Sowohl CDU als auch SPD erzielten in Ostdeutschland signifikant schlechtere Ergebnisse, während die Linkspartei/PDS dort besonders gute Resultate verzeichnete. Einen Rückgang der Wählergunst über die Zeit musste die CDU hinnehmen, wohingegen Grüne und Linkspartei zulegen konnten. Die Zugewinne der Grünen stammen aber ausschließlich aus Westdeutschland, denn in Ostdeutschland ist die Zustimmung seit 1990 gesunken. 2005 konnte die Linkspartei im Vergleich zur vorherigen Bundestagswahl in beiden Landesteilen zulegen. Ihr gelang es, unzufriedene SPD- und frühere Nichtwähler für sich zu gewinnen. ${ }^{14}$ Bei der Bundestagswahl fand eine Abkehr von den beiden Parteien statt, die einst für den Aufbau des deutschen Sozialstaats verantwortlich waren, nun diesen

13 Ausführlicher zu den kleinen Parteien: Ulrich von Alemann / Christoph Strünck, Die neue Koalitionspolitik: FDP, Bündnis 90/Die Grünen und die PDS im vereinigten Parteiensystem, in: Werner Süß (Hrsg.), Deutschland in den neunziger Jahren: Politik und Gesellschaft zwischen Wiedervereinigung und Globalisierung, Opladen 2002, S. $105-121$.

14 Vgl. Richard Hilmer / Rita Müller-Hilmer, Die Bundestagswahl vom 18. September 2005: Votum für Wechsel in Kontinuität, in: ZParl, 37. Jg. (2006), H. 1, S. 183 - 218, S. 202 f. 


\begin{tabular}{|c|c|c|c|c|c|c|}
\hline & SPD & $\begin{array}{l}\mathrm{CDU} / \\
\mathrm{CSU}\end{array}$ & $\begin{array}{l}\text { Links- } \\
\text { partei }\end{array}$ & $\begin{array}{l}\text { B'90 / } \\
\text { Grüne }\end{array}$ & FDP & Rechte \\
\hline $\begin{array}{l}\text { Region } \\
\text { (1= Ostdeutschland })\end{array}$ & $\begin{array}{l}-10,7^{* * *} \\
(2,3)\end{array}$ & $\begin{array}{l}-4,8^{*} \\
(2,3)\end{array}$ & $\begin{array}{l}11,5^{* * *} \\
(1,3)\end{array}$ & $\begin{array}{l}-0,6 \\
(0,9)\end{array}$ & $\begin{array}{l}-1,0 \\
(0,9)\end{array}$ & $\begin{array}{l}-0,8 \\
(0,4)\end{array}$ \\
\hline $\begin{array}{l}\text { Zeit } \\
\text { (Jahre seit 1990) }\end{array}$ & $\begin{array}{l}-0,1 \\
(0,1)\end{array}$ & $\begin{array}{l}-0,4^{* *} \\
(0,1)\end{array}$ & $\begin{array}{l}0,2^{* *} \\
(0,1)\end{array}$ & $\begin{array}{l}0,2^{* * *} \\
(0,1)\end{array}$ & $\begin{array}{l}-0,0 \\
(0,1)\end{array}$ & $\begin{array}{l}-0,1^{*} \\
(0,0)\end{array}$ \\
\hline Zeit* Region & $\begin{array}{c}0,4^{*} \\
(0,2)\end{array}$ & $\begin{array}{l}-0,3 \\
(0,2)\end{array}$ & $\begin{array}{c}0,2 \\
(0,1)\end{array}$ & $\begin{array}{l}-0,3^{* *} \\
(0,1)\end{array}$ & $\begin{array}{l}-0,1 \\
(0,1)\end{array}$ & $\begin{array}{l}-0,1^{* *} \\
(0,0)\end{array}$ \\
\hline Konstante & $\begin{array}{l}31,7^{* * *} \\
(1,4)\end{array}$ & $\begin{array}{l}33,7^{* * *} \\
(1,4)\end{array}$ & $\begin{array}{l}-0,3 \\
(0,8)\end{array}$ & $\begin{array}{l}4,6^{* * *} \\
(0,5)\end{array}$ & $\begin{array}{l}6,9^{* * *} \\
(0,5)\end{array}$ & $\begin{array}{l}2,0^{* * *} \\
(0,2)\end{array}$ \\
\hline korr. $\mathrm{R}^{2}$ & 0,28 & 0,44 & 0,81 & 0,41 & 0,18 & 0,13 \\
\hline \multicolumn{7}{|c|}{$\begin{array}{l}\mathrm{N}=85 \text { (= fünf Bundestagswahlen }{ }^{*} 17 \text { Länder, weil die Wahlergebnisse in Ost- und Westberlin ge- } \\
\text { trennt betrachtet werden). Rechte = DVU, Republikaner, NPD. }{ }^{*} \mathrm{p}<0,05 ;{ }^{* *} \mathrm{p}<0,01 ;{ }^{* * *} \mathrm{p}<0,001 \text {. In } \\
\text { Klammern: Standardfehler. } \\
\text { Die Interpretation des Regressionsmodells entspricht der in Tabelle } 1 . \\
\text { Quelle: Eigene Berechnung auf Grundlage der Daten des Bundeswahlleiters. }\end{array}$} \\
\hline
\end{tabular}

aber durch die Agenda 2010 oder durch die Ankündigung weiterer Einschnitte in den Augen der Wähler in Frage stellten. Als Folge konnten die beiden Volksparteien weniger Stimmen auf sich vereinen als bei allen anderen Wahlen nach 1949. Zusammen mobilisierten sie nur noch knapp 54 Prozent der Wahlberechtigten. In den siebziger Jahren waren es noch mehr als 80 Prozent gewesen.

Die Ergebnisse der Bundes- und Landtagswahlen bestätigen den Eindruck eines räumlich zweigeteilten Parteiensystems. In Ostdeutschland muss die Linkspartei/PDS als dritte Volkspartei gelten. Grüne und FDP kämpfen weiterhin darum, in den neuen Bundesländern dauerhaft Fuß zu fassen. Ausgehend von niedrigem Niveau konnte sich die SPD etwas verbessern, während der Vereinigungsbonus der CDU aufgebraucht zu sein scheint. Die Unionsparteien schnitten bei der Bundestagswahl 2005 im Gegensatz zu den ersten Jahren nach der Wiedervereinigung in den neuen Ländern deutlich schlechter $a b$ als in den alten, wo sie insbesondere im Süden erfolgreich waren.

Trotz dieser Unterschiede ist die Ost-West-Teilung nicht die einzige relevante Trennlinie. Weitere verlaufen zwischen Nord- und Süddeutschland sowie zwischen dem eher ländlichen Raum und Ballungsgebieten. Aus Tabelle 3 lässt sich ablesen, welche Faktoren für das Abschneiden der Parteien bei der Bundestagswahl 2005 ausschlaggebend waren. ${ }^{15}$ So fiel für die SPD das Nord-Süd-Gefälle stärker ins Gewicht als die Differenz zwischen West und Ost. Bündnis 90/Die Grünen gewannen die meisten Stimmen in westdeutschen Großstädten, während die Linkspartei und rechte Parteien (die NPD) überproportional gute Ergebnisse in Ostdeutschland erzielten. Auch für die FDP lag der Schwerpunkt ihrer Wahlerfolge im Westen. In Wahlkreisen mit einer relativ zum regionalen Durchschnitt höheren Arbeitslosigkeit erzielten Linkspartei und SPD überdurchschnittliche Ergebnisse, während die Arbeitslosigkeit keinen Einfluss auf das Abschneiden der NPD hatte. Einen starken, negativen Einfluss übte die Höhe der Arbeitslosigkeit auf das Wahlergebnis der FDP aus.

15 Um die Erklärungskraft der Faktoren ermessen zu können, wurden standardisierte Koeffizienten (Beta) verglichen. Sie sind in Tabelle 3 nicht extra aufgeführt. 


\begin{tabular}{|c|c|c|c|c|c|c|}
\hline \multicolumn{7}{|c|}{$\begin{aligned} \text { Tabelle 3: Einflussfaktoren auf die Wablergebnisse der Parteien in den Wablkreisen } \\
\text { bei der Bundestagswahl } 2005\end{aligned}$} \\
\hline & SPD & $\begin{array}{l}\text { CDU } / \\
\text { CSU }\end{array}$ & $\begin{array}{l}\text { Links- } \\
\text { partei }\end{array}$ & $\begin{array}{l}\text { B'90 / } \\
\text { Grüne }\end{array}$ & FDP & Rechte \\
\hline \multicolumn{7}{|l|}{ Region } \\
\hline Nord & $\begin{array}{l}2,6^{* * *} \\
(0,7)\end{array}$ & $\begin{array}{l}-0,9 \\
(0,8)\end{array}$ & $\begin{array}{l}-0,4 \\
(0,3)\end{array}$ & $\begin{array}{l}0,7 \\
(0,4)\end{array}$ & $\begin{array}{l}-0,8^{* *} \\
(0,3)\end{array}$ & $\begin{array}{c}-0,2^{*} \\
(0,1)\end{array}$ \\
\hline Ost & $\begin{array}{l}-7,7^{* * *} \\
(0,7)\end{array}$ & $\begin{array}{l}-8,5^{* * *} \\
(0,8)\end{array}$ & $\begin{array}{l}14,3^{* * *} \\
(0,3)\end{array}$ & $\begin{array}{l}-2,3^{* * *} \\
(0,4)\end{array}$ & $\begin{array}{l}-2,0^{* * *} \\
(0,3)\end{array}$ & $\begin{array}{l}1,7^{* * *} \\
(0,1)\end{array}$ \\
\hline Süd & $\begin{array}{l}-7,4^{* * *} \\
(0,6)\end{array}$ & $\begin{array}{l}5,7^{* * *} \\
(0,7)\end{array}$ & $\begin{array}{c}-0,6^{*} \\
(0,3)\end{array}$ & $\begin{array}{l}1,0^{* *} \\
(0,3)\end{array}$ & $\begin{array}{c}0,2 \\
(0,2)\end{array}$ & $\begin{array}{l}0,6^{* * * *} \\
(0,1)\end{array}$ \\
\hline Großstadt & $\begin{array}{c}0,6 \\
(0,6)\end{array}$ & $\begin{array}{l}-5,8^{* * *} \\
(0,7)\end{array}$ & $\begin{array}{l}1,0^{* * *} \\
(0,3)\end{array}$ & $\begin{array}{l}3,6^{* * *} \\
(0,3)\end{array}$ & $\begin{array}{l}-0,0 \\
(0,2)\end{array}$ & $\begin{array}{l}-0,4^{* * *} \\
(0,1)\end{array}$ \\
\hline ALQ & $\begin{array}{l}0,4^{* * *} \\
(0,1)\end{array}$ & $\begin{array}{l}-0,4^{* * *} \\
(0,7)\end{array}$ & $\begin{array}{c}0,1^{* *} \\
(0,0)\end{array}$ & $\begin{array}{l}-0,3^{* * *} \\
(0,0)\end{array}$ & $\begin{array}{l}-0,3^{* * *} \\
(0,0)\end{array}$ & $\begin{array}{c}0,0 \\
(0,0)\end{array}$ \\
\hline Konstante & $\begin{array}{c}29,6^{* * *} \\
(0,5)\end{array}$ & $\begin{array}{l}28,1^{* * *} \\
(0,6)\end{array}$ & $\begin{array}{l}3,9^{* * *} \\
(0,2)\end{array}$ & $\begin{array}{l}5,3^{* * *} \\
(0,3)\end{array}$ & $\begin{array}{l}7,9^{* * *} \\
(0,2)\end{array}$ & $\begin{array}{l}1,2^{* * *} \\
(0,1)\end{array}$ \\
\hline korr. $\mathbf{R}^{2}$ & 0,54 & 0,61 & 0,91 & 0,48 & 0,40 & 0,59 \\
\hline \multicolumn{7}{|c|}{$\begin{array}{l}\text { N = } 296 \text { (Wahlkreise). Rechte = DVU, Republikaner, NPD; Nord = Bremen, Hamburg, Niedersachsen, } \\
\text { Schleswig-Holstein; Ost = Berlin, Brandenburg, Mecklenburg-Vorpommern, Sachsen-Anhalt, Sachsen, } \\
\text { Thüringen; Süd = Baden-Württemberg, Bayern, Rheinland-Pfalz, Saarland; Referenzländer = Hessen, } \\
\text { Nordrhein-Westfalen. Großstadt = Wahlkreis mit einer Bevölkerungsdichte größer } 1000 / \mathrm{m}^{2} \text {. ALQ = } \\
\text { Abweichung vom regionenspezifischen Mittelwert der Arbeitslosigkeit. }{ }^{*} \mathrm{p}<0,05 ;{ }^{* *} \mathrm{p}<0,01 ;{ }^{* * *} \mathrm{p}<0,001 . \\
\text { In Klammern: Standardfehler. } \\
\text { Das Regressionsmodell ist wie folgt zu lesen: Für die SPD wird ein Ergebnis von } 29,6 \text { Prozent erwartet } \\
\text { (Konstante). Dieses Ergebnis erhöht sich um 2,6 Prozentpunkte, wenn der Wahlkreis in einem nord- } \\
\text { deutschen Bundesland liegt, und um zusätzliche 0,6 Prozentpunkte in Großstädten. Zudem schneidet } \\
\text { die SPD mit jedem Prozent höherer Arbeitslosigkeit um weitere 0,4 Prozentpunkte besser ab. Geschätzt } \\
\text { werden die Wahlergebnisse in Prozent der Wahlberechtigten. } \\
\text { Quelle: Eigene Berechnung auf Grundlage der Daten des Bundeswahlleiters. }\end{array}$} \\
\hline
\end{tabular}

Neben den genannten Faktoren beeinflussen Alter und Geschlecht das Wahlverhalten, wie die Repräsentative Wahlstatistik zeigt. ${ }^{16}$ Danach gaben Frauen häufiger als Männer SPD und Bündnis 90/Die Grünen ihre Stimmen, während die FDP etwas besser bei Männern abschnitt. Für die Unionsparteien zeigt sich kein ausgeprägter geschlechtsspezifischer Unterschied, wohingegen das Alter die Präferenz für CDU/CSU prägt. Die Union erreicht junge Wählerinnen und Wähler wesentlich schlechter als die über 60-Jährigen, bei denen sie im Westen weit überdurchschnittlich abschneidet. Im Gegensatz dazu erzielt die SPD ihr bestes Ergebnis bei der Gruppe der westdeutschen 18- bis 24-Jährigen. Bei der Linkspartei ist der Erfolg im Osten bei der Gruppe der 45- bis 59-Jährigen besonders groß. In dieser Altersgruppe wird sie die stärkste Partei. Die Grünen haben überdurchschnittlich viele Wähler in den Altersgruppen unter 45, die FDP bei den unter 35-Jährigen. Beide Parteien erzielen dagegen unterdurchschnittliche Ergebnisse bei älteren Wählern. Dasselbe Muster zeigt sich bei der NPD, die ihr bestes Resultat bei den ostdeutschen Männern zwi-

16 Vgl. Bundeswahlleiter, Wahl zum 16. Deutschen Bundestag am 18. September 2005. Heft 4: Wahlbeteiligung und Stimmabgabe der Männer und Frauen nach Altersgruppen, Statistisches Bundesamt, Wiesbaden 2006; vgl. auch Eckhard Jesse, Die Bundestagswahl 2005 im Spiegel der repräsentativen Wahlstatistik, in: ZParl, 37. Jg. (2006), H. 3, S. 513 ff. 
schen 18 und 24 Jahren erzielt hat. Die Volksparteien schneiden bei älteren Menschen deutlich besser ab als bei den jüngeren Altersgruppen. Betrachtet man die Verteilung der Wählerstimmen, fällt auf, dass die Volksparteien lediglich bei den über 60-Jährigen in Westdeutschland zusammen mehr als 70 Prozent der gültigen Stimmen erreichen. Während 77,4 Prozent der über 60-Jährigen angaben, für CDU/CSU oder SPD zu stimmen, lag dieser Anteil bei den 18- bis 24-Jährigen bei nur 63,3 Prozent. ${ }^{17}$ Bei jüngeren Altersgruppen und in Ostdeutschland hat sich das Parteiensystem deutlich zugunsten der kleineren Parteien verschoben. Vor diesem Hintergrund wird es zukünftig schwieriger werden, jenseits der Großen Koalition Zweiparteienbündnisse zu bilden.

Insgesamt wird deutlich, dass es neben den Unterschieden zwischen den neuen und alten Ländern eine Reihe weiterer Einflussfaktoren gibt, die sich in den Wahlergebnissen der Parteien niederschlagen. Ost- und Westdeutschland unterscheiden sich auch deshalb, weil die Parteiidentifikation in den neuen Bundesländern geringer ausgeprägt ist. ${ }^{18}$ Eine geringe Parteibindung erhöht den Anteil der Wechselwähler und führt zu höherer Volatilität. Abbildung 1 bildet diese Entwicklung bei den Bundestagswahlen mit dem Pedersen-Index $a b^{19}$, in den die Zugewinne von Parteien, Nichtwählern und Sonstigen gegenüber der vorangegangenen Wahl einfließen. Der Maximalwert von 100 Prozent würde bedeuten, dass neue Parteien bei einer Wahl alle Stimmen auf sich vereinen konnten.

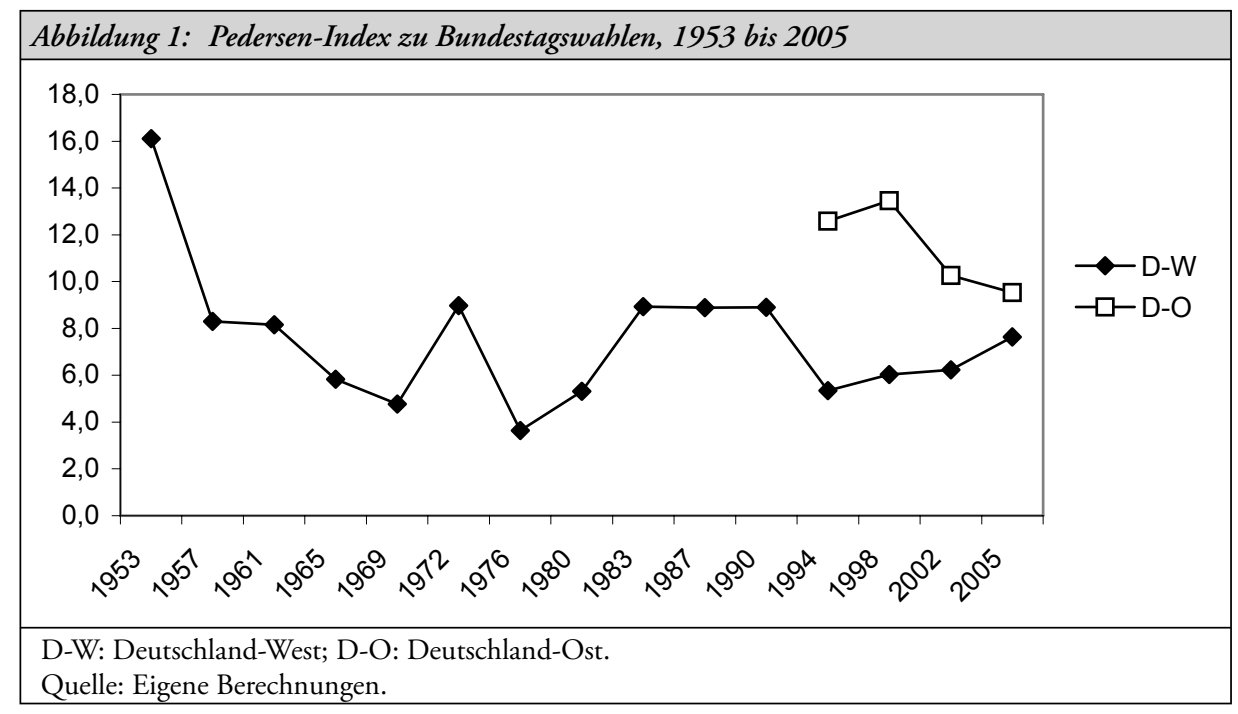

Die Werte liegen in Ostdeutschland durchweg höher als in Westdeutschland. Allerdings haben sie sich mit der letzten Bundestagswahl angenähert. Auch bei Landtagswahlen ist die Volatilität des ostdeutschen Wahlverhaltens durchschnittlich höher als im Westen, obwohl

17 Vgl. Doreen Namislo / Karina Schorn / Margitta von Schwartzenberg, Wählerverhalten bei der Bundestagswahl 2005 nach Geschlecht und Alter: Ergebnisse der Repräsentativen Wahlstatistik, in: Wirtschaft und Statistik, o. Jg. (2006), H. 3, S. 220 - 237, S. 230.

18 Ausführlich dazu: Kai Arzheimer / Jürgen W. Falter, „Goodbye Lenin?“, a.a.O., S. 161 - 171.

19 Mogens N. Pedersen, The Dynamics of European Party Systems: Changing Patterns of Electoral Volatility, in: European Journal of Political Research, 7. Jg. (1979), S. 1 - 26. 
es in beiden Regionen erhebliche Abweichungen gibt. Die höhere Volatilität bei Landtagswahlen in Ostdeutschland wird vor allem durch die rückläufige Wahlbeteiligung bestimmt. ${ }^{20}$ Allerdings nimmt die Beteiligung bei westdeutschen Wahlen inzwischen schneller als im Osten ab, weshalb eine weitere Annäherung der Volatilität zu erwarten ist.

Bei der Wahlbeteiligung geben die neuen Länder das Muster vor, dem die alten folgen. Der schnelle und anhaltende Rückgang bei Landtagswahlen ist eine der eindrücklichsten Veränderungen im politischen System Deutschlands. Zwischen 1946 und Ende der achtziger Jahre lag die durchschnittliche Beteiligung bei Landtagswahlen bei etwa 80 Prozent, und es gab keine Anzeichen wachsender Wahlmüdigkeit. Seit 1990 geht die Wahlbeteiligung kontinuierlich zurück. In westdeutschen Bundesländern fiel sie in den vergangenen anderthalb Jahrzehnten von durchschnittlich 76 auf 57, in Ostdeutschland von knapp 70 auf 52 Prozent. Dieser Trend hat alle Bundesländer erfasst. Nirgends liegt die Wahlbeteiligung heute höher oder auf gleichem Niveau wie Ende der achtziger Jahre. Ähnliche Entwicklungen lassen sich bei den Kommunal- und Europawahlen beobachten. Lag die durchschnittliche Wahlbeteiligung bei Kommunalwahlen in den siebziger Jahren noch knapp über 80 Prozent, fiel sie in den Achtzigern auf 75, in den Neunzigern auf 67 und in den Zweitausendern auf 56 Prozent. Mit leichten Schwankungen haben sich die Wahlen zum Europäischen Parlament in die gleiche Richtung bewegt. Konnten anfangs noch um die 60 Prozent der Wähler mobilisiert werden, waren es 1999 und 2004 nur noch 45 beziehungsweise 43 Prozent der Wahlberechtigten. ${ }^{21}$ Auf Bundesebene ist ebenfalls ein rückläufiger Trend erkennbar. In den elf Bundestagswahlen zwischen 1949 und 1987 lag die durchschnittliche Wahlbeteiligung bei 87 Prozent, in den fünf Wahlen seit 1990 bei 79 Prozent. An der Bundestagswahl 2005 nahmen weniger Wähler als jemals zuvor teil. In Übereinstimmung mit der Entwicklung in anderen westeuropäischen Ländern sinkt die Wahlbeteiligung auf allen Ebenen des politischen Systems. ${ }^{22}$

Zusammenfassend lässt sich festhalten, dass weiterhin erkennbare Unterschiede im Wahlverhalten in Ost- und Westdeutschland bestehen. Die aggregierte Volatilität ist in den

20 Unterzieht man die Mittelwerte des Pedersen-Index für ost- und westdeutsche Landtagswahlen einem statistischen Vergleich, ergeben sich überzufällige Unterschiede $(\mathrm{N}=56$; $\mathrm{df}=40,384$; $\mathrm{t}=-3,244)$. Wird der Volatilitäts-Index jedoch ohne Berücksichtigung der Nichtwähler konstruiert, ist die Mittelwertsdifferenz nicht länger statistisch signifikant $(\mathrm{N}=56 ; \mathrm{df}=42,004 ; \mathrm{t}=-1,995)$.

$21 \mathrm{Ob}$ eine niedrige Wahlbeteiligung ein Problem für die Legitimation der Politik darstellt, ist in der Literatur umstritten. Würde sich die Wahlapathie gleichmäßig über alle Bevölkerungsschichten verteilen, wäre sie weniger bedenklich. Doch stattdessen gilt: „Niedrige Wahlbeteiligung ist generell ungleiche Wahlbeteiligung“, so Ulrich Kohler, Die soziale Ungleichheit der Wahlabstinenz in Europa, in: Jens Alber / Wolfgang Merkel (Hrsg.), Europas Osterweiterung: Das Ende der Vertiefung?, WZB-Jahrbuch 2005, Berlin 2006, S. 159 - 179, S. $170 \mathrm{f}$.

22 Vgl. Peter Mair, Ruling the Void? The Hollowing of Western Democracy, in: New Left Review, 42. Jg. (2006), S. 25 - 51. An dieser Stelle kann keine Analyse der Nichtwahl vorgenommen werden; vgl. dazu Norbert Kersting, Nichtwähler: Diagnose und Therapieversuche, in: ZPol, 14. Jg. (2004), S. 403 - 427. Dennoch soll auf einige Umfrageergebnisse hingewiesen werden. Eine überwältigende Mehrheit der Bevölkerung begreift die Teilhabe an politischen Entscheidungen als demokratisches Grundrecht. Die Möglichkeit, tatsächlich Einfluss zu nehmen, wird jedoch als gering angesehen. 72,4 Prozent der Befragten stimmen der Aussage „was ich denke, kümmert die Regierung nicht viel“ zu, und 61,8 Prozent denken, dass „Menschen wie ich ... nichts zu sagen" haben. 62,8 Prozent meinen, dass die Parteien keine Alternativen bieten, und mehr als die Hälfte der Befragten glaubt, dass Politiker vor allem wegen ihres persönlichen Vorteils in der Politik sind (Daten: ALLBUS 2004, ZA-Nr. 3762). 
fünf neuen Ländern höher, die Wahlbeteiligung niedriger. Allerdings sinkt auch im Westen die Wahlbeteiligung, bei Landtagswahlen sogar schneller als im Osten. Rückläufige Parteibindungen haben den Übergang vom Zweieinhalb- zum „fluiden Fünfparteiensystem“23 erleichtert und die Volksparteien geschwächt. Dies ging nach der Wiedervereinigung mit einer Linksverschiebung des parlamentarischen Gravitationszentrums einher. Die Unterschiede zwischen den Landesteilen führen zu abweichenden Mustern der Koalitionsbildung.

\section{Koalitionen im Bund und in den Ländern}

Aus den 69 Landtagswahlen zwischen 1990 und 2007 sind zehn unterschiedliche Regierungstypen hervorgegangen. Neben der Alleinregierung einer der beiden Volksparteien führten die Wahlen zu acht Koalitionsvarianten. An fünf davon ist die SPD beteiligt, an dreien die CDU. Dies liefert einen ersten Hinweis auf die koalitionsstrategisch günstigere Ausgangslage der Sozialdemokratie. Die häufigste Regierung in den Bundesländern ist eine Große Koalition, weil in Ostdeutschland in fast einem Drittel der Fälle CDU und SPD gemeinsam regieren. In westdeutschen Bundesländern ist dies weit seltener der Fall (zwölf Prozent). Diese Differenz geht auf das unterschiedliche Abschneiden der kleinen Parteien in Ost- und Westdeutschland zurück. Während die Linkspartei/PDS bei allen Wahlen in den fünf neuen Ländern und in Berlin den Sprung in den Landtag schaffte, ist ihr dies im Westen erst einmal gelungen. Die Grünen sind dagegen in westdeutschen Landtagen und Berlin fest etabliert. In 43 von 47 Wahlen übersprangen sie die Fünfprozenthürde. In Ostdeutschland ist ihre parlamentarische Verankerung dagegen schwach. Nur in sechs von 22 Wahlen erhielten Bündnis 90/Die Grünen mehr als fünf Prozent der gültigen Stimmen. Vier dieser Erfolge wurden 1990 bei den ersten Landtagswahlen in Ostdeutschland erzielt. In jüngster Zeit gelang nur in Sachsen der Einzug in ein Landesparlament. Für die FDP galt lange Zeit ein ähnliches Muster, wobei sie im Westen schlechter (in elf von 47 Wahlen misslang der Einzug ins Parlament), im Osten jedoch besser als die Grünen abgeschnitten hat. 2007 ist die FDP in drei ostdeutschen Landtagen vertreten.

Aus dieser Konstellation ergibt sich für die CDU die Schwierigkeit, passende Koalitionspartner zu finden, obwohl sie im Durchschnitt aller Landtagswahlen seit 1990 bessere Ergebnisse als die SPD erzielt hat. Lässt man Große Koalitionen außen vor, sind jedoch die Sozialdemokraten an 56 Prozent, die Christdemokraten an 44 Prozent der Landesregierungen beteiligt. Die SPD hat zudem mit allen im Bundestag vertretenen Parteien auf Landesebene koaliert. Die CDU muss dagegen entweder eine absolute Mehrheit in den Parlamenten erringen oder ist auf die Zusammenarbeit mit der FDP angewiesen, die jedoch gerade in Ostdeutschland relativ schwach ist. Sonst bleibt ihr nur das Bündnis mit der SPD. Der regelmäßig wiederkehrende Flirt mit den Grünen erklärt sich aus dem Versuch, weitere Koalitionsmöglichkeiten zu entwickeln. ${ }^{24} \mathrm{Da}$ Landesregierungen häufig der Testfall

23 Vgl. Oskar Niedermayer, Das Parteiensystem Deutschlands, in: ders. / Richard Stöss / Melanie Haas (Hrsg.), Die Parteiensysteme Westeuropas, Wiesbaden 2006, S. 109 - 133, S. 130.

24 Diese Avancen treffen auf Zustimmung bei der grünen Parteiführung. So zeigte sich Reinhard Bütikofer in einem Zeitungsinterview aufgeschlossen gegenüber schwarz-grünen Bündnissen auf Landesebene und einer möglichen „Jamaika“-Koalition im Bund, in: Passauer Neue Presse vom 15. März 2007. 
für eine spätere bundespolitische Kooperation sind, hat die SPD hier einen klaren Vorsprung gegenüber der CDU.

In Ostdeutschland besteht zudem eine strukturelle Asymmetrie zugunsten der Parteien links der Mitte. SPD, Linkspartei/PDS und Grüne konnten bei Landtagswahlen (Ostdeutschland und Ostberlin) im Schnitt 33,3 Prozent der Wahlberechtigten mobilisieren. Die bürgerlichen Parteien (CDU und FDP) erreichten dagegen nur 24,1 Prozent der Wähler. ${ }^{25}$ In den ersten Jahren nach 1990 scheuten die Sozialdemokraten zwar eine Zusammenarbeit mit der PDS, doch mit der Duldung erst einer rot-grünen, dann einer SPDMinderheitsregierung durch die PDS in Sachsen-Anhalt - das „Magdeburger Modell“ verschwanden diese Hemmungen. Regierungskoalitionen beider Parteien folgten in Mecklenburg-Vorpommern und Berlin. Bei den Landtagswahlen im Westen zeigt sich, dass SPD und Grüne sowie CDU und FDP im Schnitt gleichauf liegen (30,3 beziehungsweise 30,9 Prozent der Wahlberechtigten).

Während die Sozialdemokraten nach dem Zweiten Weltkrieg schon früh in westdeutschen Landtagen Erfolge feierten und in einigen norddeutschen Bundesländern dauerhaft Regierungspartei wurden, dominierte auf Bundesebene die CDU. Richard Stöss und Gero Neugebauer verweisen darauf, dass die Union "gewissermaßen von Natur aus die stärkste Partei ist“, da „die soziale Basis der Unionsparteien größer als die der SPD ist “26. Dementsprechend erzielte die SPD nur bei drei Bundestagswahlen, 1972, 1998 und 2002, mehr Stimmen als die Unionsparteien. Niedermayer sieht das westdeutsche Parteiensystem vor der Vereinigung durch eine strukturelle bürgerliche Mehrheit charakterisiert. ${ }^{27}$ In den späten sechziger und siebziger Jahren waren jedoch alle Parteien bereit, miteinander zu koalieren. Dies erhöhte die Bedeutung der Freidemokraten, da sie nun der Königsmacher waren und diese Rolle gekonnt ausspielten. Seit Anfang der achtziger Jahre verstärkte die FDP ihren marktwirtschaftlichen Kurs, der 1982 dazu führte, dass sie das konstruktive Misstrauensvotum gegen Helmut Schmidt unterstützte und seitdem ideologisch der CDU näher steht. Durch die Wiedervereinigung haben sich die Gewichte von den bürgerlichen Parteien zugunsten des Mitte-links-Blocks verschoben. Dies ist vornehmlich in Ostdeutschland der Fall, wo nach einem Übergewicht von CDU und FDP bei der Bundestagswahl 1990 seither SPD und Linkspartei/PDS zulegen konnten. Während CDU/CSU/FDP und SPD/Grüne/Linke im Westen fast gleichauf liegen, konnten die drei linken Parteien in Ostdeutschland bei den letzten drei Bundestagswahlen jeweils mehr als 60 Prozent der gültigen Stimmen erringen, CDU und FDP gemeinsam dagegen nur knapp ein Drittel. Lautete 1998 die berechtigte Frage, ob der rot-grüne Wahlsieg ein einmaliger Ausreißer bleiben würde ${ }^{28}$, hat es seither im Bundestag immer eine Mehrheit links der Unionsparteien gegeben.

25 Die linke Mehrheit bleibt auch dann bestehen, wenn man die Grünen nicht beachtet und Ostberlin außen vor lässt, wo die Linkspartei/PDS überdurchschnittlich stark abgeschnitten hat.

26 Richard Stöss / Gero Neugebauer, Die SPD und die Bundestagswahl 1998: Ursachen und Risiken eines historischen Wahlsiegs unter besonderer Berücksichtigung der Verhältnisse in Ostdeutschland, Arbeitshefte aus dem Otto-Stammer-Zentrum Nr. 2, Freie Universität Berlin 1998, S. 7.

27 Oskar Niedermayer, a.a.O. (Fn. 23), S. 115 f.

28 Ulrich von Alemann, Der Wahlsieg der SPD von 1998: Politische Achsenverschiebung oder glücklicher Ausreißer?, in: Oskar Niedermayer (Hrsg.), Die Parteien nach der Wahl 1998, Opladen 1999, S. 37 - 62. 
Tabelle 4: Theoretisch mögliche „minimale Gewinnkoalitionen“ im Bundestag, 1980 bis 2005

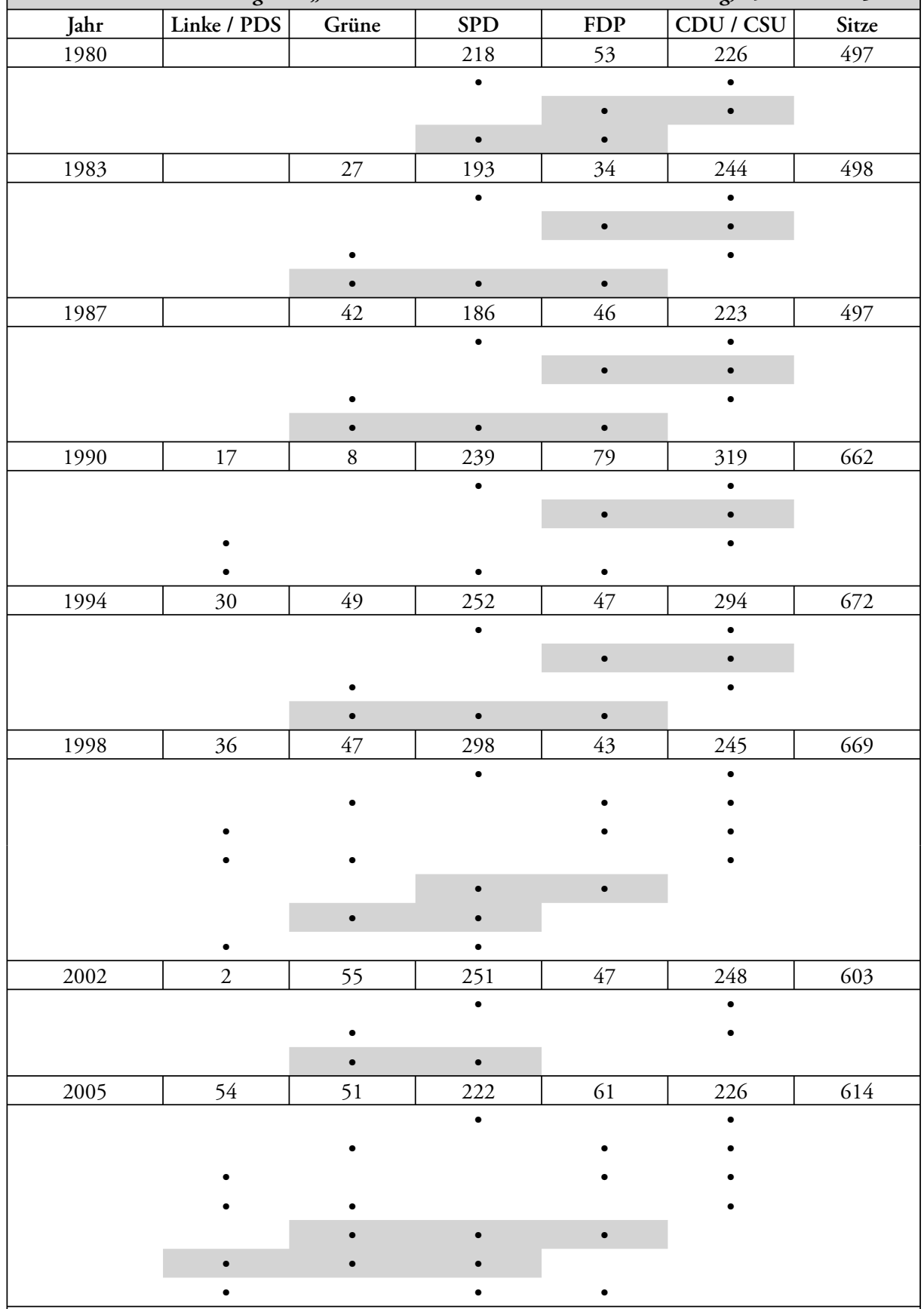

Grau hinterlegte Koalitionsvarianten bezeichnen ideologisch verbundene minimale Gewinnkoalitionen.

Quelle: Eigene Berechnungen. 
In Tabelle 4 sind alle theoretisch möglichen „minimalen Gewinnkoalitionen“ (MGK) seit 1980 durch schwarze Punkte gekennzeichnet. Eine minimale Gewinnkoalition wird dadurch definiert, dass jede der Koalitionsfraktionen für das Erreichen einer parlamentarischen Mehrheit benötigt wird. ${ }^{29}$ Die grau hinterlegten Felder der Tabelle geben zusätzlich an, welche dieser MGK ideologisch verbunden sind. Dem liegt die Annahme zugrunde, dass „verbundene“ Parteien mehr Gemeinsamkeiten aufweisen als unverbundene und ihnen deshalb eine Zusammenarbeit leichter gelingt. ${ }^{30}$ Die Rangfolge der Parteien entspricht ihrer Verortung auf der Links-rechts-Achse. ${ }^{31}$ Noch 1980 gab es lediglich drei minimale Gewinnkoalitionen. Zwei davon waren verbundene minimale Gewinnkoalitionen und an beiden war die FDP beteiligt. Dies entsprach im Wesentlichen der Situation des Zweieinhalbparteiensystems der Bundesrepublik und erklärt, weshalb es den Freidemokraten gelang, länger als jede andere Partei an der Regierung beteiligt zu sein.

Nach dem Einzug der Grünen in den Bundestag 1983 entstanden weitere theoretische Koalitionsmöglichkeiten, die jedoch entweder ideologisch unverbunden waren oder ein Dreiparteienbündnis erfordert hätten. 1990 gewannen Bündnis 90/Die Grünen, 1994 die PDS jeweils zu wenig Sitze, um für irgendeine minimale Gewinnkoalition benötigt zu werden. Da auch die SPD bei beiden Wahlen deutlich hinter dem Ergebnis der Unionsparteien zurückblieb, war bis 1994 die christlich-liberale Koalition die einzig mögliche verbundene MGK. Dies änderte sich 1998, als die SPD stärkste im Bundestag vertretene Fraktion wurde. Insgesamt gab es nun sieben theoretische MGK, doch nur zwei davon waren ideologisch verbunden. Die Sozialdemokraten konnten wählen, ob sie mit der FDP oder den Grünen regieren wollten, und entschieden sich für die ihnen ideologisch näherstehenden Grünen. Diese Koalition wurde 2002 fortgeführt. In dieser Wahlperiode waren weder die PDS, die nur noch mit zwei direkt gewählten Abgeordneten im Bundestag vertreten war, noch die FDP für die Koalitionsbildung relevant. Für die CDU stand eine Große Koalition oder ein Bündnis mit den Grünen zur Auswahl.

Die Bundestagswahl 2005 durchbrach das seit Abschluss der Konsolidierungsphase des deutschen Parteiensystems 1961 bestehende Muster möglicher Koalitionen. Zum ersten Mal gab es keine verbundene minimale Gewinnkoalition aus nur zwei Parteien. Die einzigen theoretisch verfügbaren verbundenen MGK bestanden aus einem Dreierbündnis von FDP, SPD und Grünen oder aus einem Linksbündnis von SPD, Grünen und Linkspartei. Da jedoch die Linkspartei jegliche Regierungsbeteiligung ausschloss und die FDP nicht zu einer Koalition unter Führung der SPD bereit war, blieb nur das Bündnis der beiden Volksparteien. Deren Schwäche und das Unbehagen an der Großen Koalition könnte künftig die Suche nach neuen Bündnissen intensivieren. Die Sozialdemokraten verfügen hierbei über mehr Optionen als die Christdemokraten, falls es diesen nicht in einem ersten Schritt gelingt, auf Landesebene neue Koalitionen einzugehen.

29 William H. Riker, The Theory of Political Coalitions, New Haven / London 1962, S. 40.

30 Robert Axelrod, Conflict of Interest: A Theory of Divergent Goals with Applications to Politics, Chicago 1970, S. 170.

31 Die ideologische Verortung der Parteien ist umstritten und hängt von den betrachteten Issues ab. Hier wird in der Rangfolge einer Reihe von Expertenbefragungen gefolgt (Chapel Hill Party Dataset 2002), die Parteien in 23 Ländern zu verschiedenen Zeitpunkten (1984, 1988, 1992, 1996, 1999 und 2003) auf einer allgemeinen Links-rechts-Skala verortet haben. Auch wenn sich Werte für die einzelnen Parteien im Zeitverlauf verändern - CDU und SPD nähern sich ideologisch an -, bleibt doch die Reihenfolge gleich. 


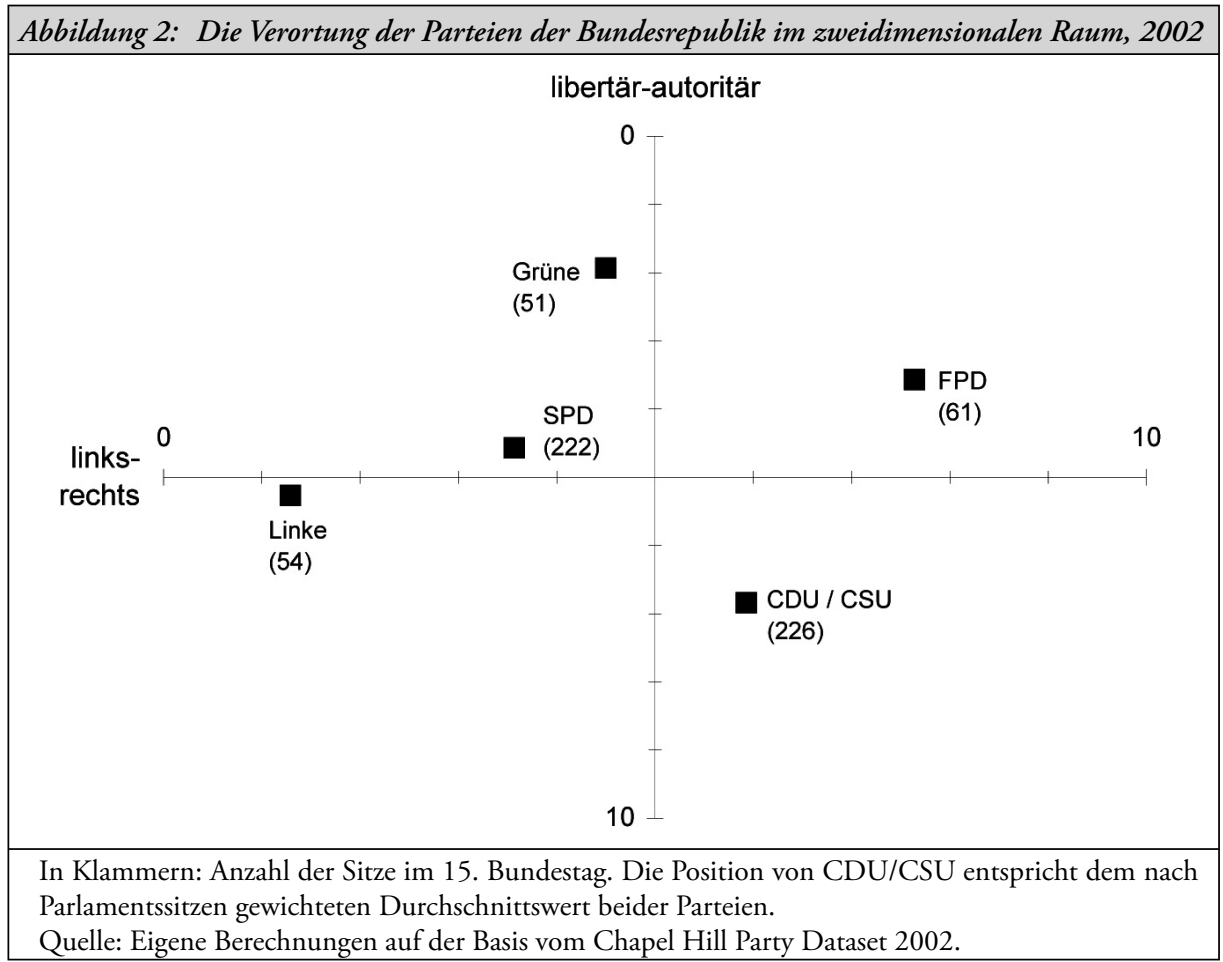

Abbildung 2 verortet die im Bundestag vertretenen Parteien in einem zweidimensionalen Raum, dessen Achsen die libertär-autoritäre sowie die ökonomische Links-rechts-Achse bilden. Daraus lassen sich erneut die minimalen Gewinnkoalitionen ablesen. Da nun nicht länger eine allgemeine Links-rechts-Skala verwendet wird, ergeben sich etwas andere Koalitionsmuster. Auf der ökonomischen Achse bestehen zwei denkbare Bündnisse: einerseits die „Jamaika-Koalition“ und andererseits die Linkskoalition aus SPD, Grünen und Linkspartei. Nimmt man stattdessen die gesellschaftspolitische Dimension in den Blick, existieren zwei verbundene MGK: zum einen SPD, FDP und Grüne, zum anderen die - faktisch ausgeschlossene - Koalition aus SPD, Linkspartei und FDP. Bemerkenswert daran ist, dass die Grünen, obwohl sie die kleinste Fraktion im Bundestag stellen, in drei theoretisch möglichen Bündnissen vorkommen. Bei den beiden Volksparteien hat die SPD die bessere Ausgangsposition, da sie Partner in drei denkbaren Koalitionen ist, die Union dagegen nur in einer.

Dieses Ergebnis wird bestätigt, wenn man den durchschnittlichen Abstand (die so genannte euklidische Distanz) zwischen den Koordinaten einer Partei und denen der jeweils vier anderen berechnet. Den geringsten Wert weist die SPD $(3,2)$ auf, gefolgt von den Grünen $(4,0)$, den Unionsparteien $(4,2)$, der FDP $(4,5)$ und schließlich der Linkspartei $(4,6)$. Allerdings beruht diese formale Betrachtung auf der Annahme, dass die Dimensionen gleich gewichtet sind. Realistischer ist, dass es hierbei Unterschiede zwischen den Parteien gibt. Dennoch lässt sich festhalten, dass gegenwärtig die SPD den zentralen Platz im deutschen Parteiensystem einnimmt und nicht nur theoretisch, sondern auch in der Praxis über die meisten Koalitionsoptionen verfügt. Sie hat mit allen im Bund vertretenen Parteien 
bereits auf Landesebene koaliert und könnte sowohl eine Ampel-Koalition als auch ein Linksbündnis anführen. Solange es der CDU nicht gelingt, mit den Grünen zu koalieren, bleiben ihre Optionen auf FDP und SPD begrenzt. Diese parteipolitische Konstellation begründet gegenwärtig die relative Stärke der Sozialdemokratie.

\section{Sozialstaatsbefürworter in Ost und West}

Im Wahlkampf 2005 erklärte Edmund Stoiber, er akzeptiere nicht, „dass der Osten bestimmt, wer in Deutschland Kanzler wird. Die Frustrierten dürfen nicht über Deutschlands Zukunft bestimmen“32. Aus dieser Äußerung sprach die Einsicht, dass die Enttäuschten im Osten sich von den Unionsparteien abgewandt haben. Auch in der Wissenschaft gibt es Beobachter, die eine „Versorgungsmentalität“ und einen Mangel „an bürgerlichen Lebensweisen und Leitbildern“ im Osten sehen. Schneidet die CDU wie in Sachsen bei Wahlen gut ab, wird dies als Ausweis für einen erfolgreichen „Mentalitätswandel“ gesehen ${ }^{33}$. Im Gegensatz dazu wurde oben auf den für alle Landesteile geltenden Zusammenhang zwischen der Höhe der Arbeitslosigkeit und dem Abschneiden von SPD und Linkspartei verwiesen (siehe Tabelle 3). Anstatt einen vermeintlichen Modernitätsrückstand der Wähler im Osten zu beklagen, soll hier der Einstellung der Bürger zum Sozialstaat nachgegangen werden.

Die deutschen Wähler stehen mit ihren Sozialstaatspräferenzen mehrheitlich links der Volksparteien. In Meinungsumfragen sprechen sich 70 Prozent der Befragten gegen weitere Kürzungen im Sozialsystem aus. Gleichzeitig befürworten ebenfalls 70 Prozent eine stärkere Besteuerung höherer Einkommen ${ }^{34}$. Mehr als die Hälfte der Bevölkerung wünscht sich mehr Absicherung in der sozialen Marktwirtschaft, nur ein Drittel fordert eine Stärkung des Markts ${ }^{35}$. Ebenso sieht eine große Mehrheit die zur Haushaltskonsolidierung ergriffenen Maßnahmen kritisch. Jeweils über 70 Prozent der Befragten lehnen die Kürzung der Pendlerpauschale und der Sparerfreibeträge sowie die Erhöhung der Mehrwertsteuer ab. Aber 76 Prozent unterstützen eine „Reichensteuer“ 36 . Einen längeren Bezug von Arbeitslosengeld für diejenigen, die länger Beiträge gezahlt haben, befürworten 82 Prozent der Wähler ${ }^{37}$. Dagegen sprechen sich 83 Prozent gegen die „Rente ab 67“ aus ${ }^{38}$. Diese Liste ließe sich fortsetzen. Zugrunde liegt das Gefühl, dass die Problemlösungsfähigkeit der Politik gering ist und eine Gerechtigkeitslücke besteht. Zwei Drittel der Bevölkerung sind der Meinung, dass es in Deutschland „eher ungerecht“ zugeht, 72 Prozent empfinden das Rentensystem, 74 Prozent den Umgang mit Schwachen und 81 Prozent das Steuersystem als ungerecht $^{39}$.

32 Frankfurter Allgemeine Zeitung vom 11. August 2005.

33 Alle Zitate aus Eckhard Jesse, Parteiensystem im Wandel? Das deutsche Parteiensystem vor und nach der Bundestagswahl 2005, in: ders. I Roland Sturm (Hrsg.), Bilanz der Bundestagswahl 2005, a.a.O., S. $21-41$, S. 33 f.

34 Forschungsgruppe Wahlen, Politbarometer vom 24. Juni 2005.

35 Forschungsgruppe Wahlen, Politbarometer vom 10. November 2006.

36 Forschungsgruppe Wahlen, Politbarometer vom 19. Mai 2006.

37 Forschungsgruppe Wahlen, Politbarometer vom 10. November 2006.

38 Forschungsgruppe Wahlen, Politbarometer vom 2. März 2007.

39 Infratest dimap, ARD DeutschlandTREND, Dezember 2006. 
Wird untersucht, welche Art von Sozialstaat die Deutschen bevorzugen, spricht sich eine Mehrheit für ein umfassendes System sozialer Sicherung aus. Die Forschung hat „drei Welten des Wohlfahrtskapitalismus“ identifiziert: den liberalen, den christdemokratischen und den sozialdemokratischen Sozialstaat. ${ }^{40}$ Diese unterscheiden sich hinsichtlich der Frage, wie sehr das Einkommen einer Person vom am Markt erzielbaren Lohn abhängt und welche Verantwortung der Staat bei der Absicherung sozialer Risiken hat. Edeltraud Roller analysiert anhand von Umfragedaten des International Social Survey Programme, welchen Sozialstaatstyp die Deutschen mehrheitlich unterstützen. ${ }^{41}$ Sie fügt Gøsta Esping-Andersens Typologie ein viertes, „sozialistisches“ Sozialstaatsmodell hinzu, das sich von der sozialdemokratischen Variante dadurch unterscheidet, dass die staatliche Verantwortung nicht nur Einkommenssicherheit in Risikofällen, Chancengleichheit, Ergebnisgleichheit und Vollbeschäftigung, sondern darüber hinaus die Kontrolle der Löhne und Gehälter umfasst. 1996 befürworteten 57 Prozent der befragten Westdeutschen den sozialdemokratischen und 26 Prozent den sozialistischen Sozialstaatstyp. In Ostdeutschland sprachen sich 68 Prozent für das sozialistische und 27 Prozent für das sozialdemokratische Modell aus. Es weisen also nicht nur Ostdeutsche eine ausgeprägte (Sozial-)Staatsorientierung auf, sondern auch die Westdeutschen fordern eine umfassende soziale Verantwortung des Staats.

Dieser positiven Grundhaltung steht ein akutes Krisenempfinden bezüglich der sozialen Sicherungssysteme gegenüber. ${ }^{42}$ Knapp 50 Prozent der Ostdeutschen und 42 Prozent der Westdeutschen glauben, dass deren Zusammenbruch kurz bevorstehe. Der Rest sieht „größere Probleme“, während in beiden Landesteilen nur eine winzige Minderheit davon ausgeht, dass „alles in Ordnung“ sei. Ebenso einhellig sind mehr als neunzig Prozent aller Befragten der Meinung, dass in Deutschland das Problem der Arbeitslosigkeit noch lange Zeit bestehen wird. Angesichts dieser Einschätzung ist eine Mehrheit in Ost- und Westdeutschland bereit, zum Beispiel durch Lohnverzicht einen Beitrag zur Sicherung der Arbeitsplätze zu leisten. Dagegen lehnt eine Mehrheit in beiden Landesteilen eine Lockerung des Kündigungsschutzes ab. Nach den Reformen der rot-grünen Bundesregierung befragt, antwortete 2004 eine knappe Mehrheit der Westdeutschen (51 Prozent), dass diese „eher richtig“ gewesen seien. In Ostdeutschland überwog die Ablehnung (55 Prozent). In beiden Landesteilen bestand eine ausgesprochene Skepsis hinsichtlich der Effektivität der eingeleiteten Reformen. In West wie Ost bezweifelten mehr als 80 Prozent der Befragten, dass die beschlossenen Maßnahmen bestehende Probleme lösen würden. Wird direkt gefragt, ob Sozialleistungen gekürzt, beibehalten oder ausgeweitet werden sollen, verteidigt eine (relative) Mehrheit den Status quo (West: 57; Ost: 48 Prozent). Eine Ausweitung der Sozialleistungen befürworten 17 Prozent der Westdeutschen und 42 Prozent der Ostdeutschen. Nur jeder Vierte im Westen und jeder Zehnte im Osten spricht sich für eine Kürzung von Sozialleistungen aus. ${ }^{43}$

40 Gøsta Esping-Andersen, The Three Worlds of Welfare Capitalism, Cambridge 1990.

41 Edeltraud Roller, Ende des sozialstaatlichen Konsenses? Zum Aufbrechen traditioneller und zur Entstehung neuer Konfliktstrukturen in Deutschland, in: Oskar Niedermayer / Bettina Westle (Hrsg.), Demokratie und Partizipation: Festschrift für Max Kaase, Wiesbaden 2000, S. 88 114.

42 Alle Angaben dieses Absatzes entstammen dem Politbarometer Ost 2004 (ZA-Nr. 4221) sowie dem Politbarometer West 2004 (ZA-Nr. 4222).

43 ALLBUS 2004, Za-Nr. 3762. 


\begin{tabular}{|c|c|c|c|}
\hline Aussage: & D-W & D-O & $\mathrm{t}$-Wert \\
\hline $\begin{array}{l}\text { 1) „Das Einkommen sollte sich nicht allein nach der Leistung des Einzelnen } \\
\text { richten. Vielmehr sollte jeder das haben, was er für sich und seine Familie } \\
\text { für ein anständiges Leben braucht.“ }\end{array}$ & 40 & 49 & $\begin{array}{c}4,3^{* * *} \\
(\mathrm{~N}=2853)\end{array}$ \\
\hline $\begin{array}{l}\text { 2) „Der Staat muss dafür sorgen, dass man auch bei Krankheit, Not, } \\
\text { Arbeitslosigkeit und im Alter ein gutes Auskommen hat.“ }\end{array}$ & 83 & 92 & $\begin{array}{c}7,8^{* * *} \\
(\mathrm{~N}=2914)\end{array}$ \\
\hline $\begin{array}{l}\text { 3) „Der Staat muss dafür sorgen, dass jeder Arbeit hat und die Preise stabil } \\
\text { bleiben, auch wenn deswegen die Freiheiten der Unternehmer einge- } \\
\text { schränkt werden müssen.“ }\end{array}$ & 64 & 77 & $\begin{array}{c}7,5^{* * *} \\
(\mathrm{~N}=2855)\end{array}$ \\
\hline $\begin{array}{l}\text { 4) „Ich finde die sozialen Unterschiede in unserem Land im Großen und } \\
\text { Ganzen gerecht.“ }\end{array}$ & 35 & 16 & $\begin{array}{c}12,2 * * * \\
(\mathrm{~N}=2845)\end{array}$ \\
\hline $\begin{array}{l}\text { 5) „Wenn die Leistungen der sozialen Sicherung, wie Lohnfortzahlungen im } \\
\text { Krankheitsfall, Arbeitslosenunterstützung und Frührenten so hoch sind } \\
\text { wie jetzt, führt dies nur dazu, dass die Leute nicht mehr arbeiten wollen.“ }\end{array}$ & 63 & 47 & $\begin{array}{c}8,2^{* * *} \\
(\mathrm{~N}=2815)\end{array}$ \\
\hline $\begin{array}{l}\text { 6) „Nur wenn die Unterschiede im Einkommen und im Ansehen groß } \\
\text { genug sind, gibt es auch einen Anreiz für persönliche Leistungen.“ }\end{array}$ & 57 & 35 & $\begin{array}{c}11,4^{* * *} \\
(\mathrm{~N}=2835)\end{array}$ \\
\hline \multicolumn{4}{|c|}{$\begin{array}{l}{ }^{*} \mathrm{p}<0,05 ;{ }^{* *} \mathrm{p}<0,01 ;{ }^{* * *} \mathrm{p}<0,001 \text {. D-W: Deutschland-West; D-O: Deutschland-Ost. } \\
\text { Angegeben ist der Prozentsatz der Befragten, die der Aussage zustimmen. Dies umfasst „stimme eher } \\
\text { zu“ und „stimme voll zu“. Die Ablehnung (,stimme eher nicht zu“ und ,stimme überhaupt nicht zu“) } \\
\text { entspricht der Differenz des angeführten Werts von } 100 \text { Prozent. Überprüft wurde, ob die Abweichung } \\
\text { der Zustimmungsraten überzufällig ist. } \\
\text { Quelle: Eigene Berechnungen auf Basis der Daten vom ALLBUS } 2004 \text {, ZA-Nr. } 3762 \text {. }\end{array}$} \\
\hline
\end{tabular}

Tabelle 5 verdeutlicht Gemeinsamkeiten und Unterschiede in der Einstellung zum Sozialstaat. Dabei zeigen sich widersprüchliche Befunde. Eine (erhebliche) Minderheit stimmt der Aussage zu, dass das Einkommen sich nicht allein an die Leistung, sondern an den Bedürfnissen orientieren sollte (Aussage 1). Dem Staat wird von einer überwältigenden Mehrheit in beiden Landesteilen die Aufgabe zugeschrieben, die Menschen gegen soziale Risiken abzusichern (2). Mehr als zwei Drittel der Befragten sehen den Staat darüber hinaus in der Verantwortung für stabile Preise und Arbeitsplätze (3). Jeweils eine Mehrheit in Ost und West findet die bestehende Ungleichheit in Deutschland nicht gerecht (4). Gleichzeitig ist allerdings eine Mehrheit der Westdeutschen überzeugt, dass großzügige Sozialleistungen die Arbeitsmotivation mindern (5) und dass Einkommens- und Ansehensunterschiede einen persönlichen Leistungsanreiz bieten (6). In Ostdeutschland stimmt die Mehrheit beiden Aussagen nicht zu. Diese Daten verweisen bei aller Widersprüchlichkeit darauf, dass die staatliche Absicherung sozialer Risiken und statuserhaltende Sicherungssysteme große Zustimmung finden. Strittig dagegen sind Maßnahmen, die Umverteilung zum Ziel haben. ${ }^{4}{ }^{4}$ Das Auskommen, das der Sozialstaat gewährt, muss man sich zumindest in den Augen der Westdeutschen verdient haben. Obwohl also eine positive Grundhaltung zum Sozialstaat in beiden Landesteilen besteht, sprechen die Durchschnittswerte für eine stärker ausgeprägte Sozialstaatsorientierung der Ostdeutschen.

44 Carsten G. Ullrich, Die soziale Akzeptanz des Wohlfahrtsstaates: Anmerkungen zum Forschungsstand, in: Arbeitspapiere - Mannheimer Zentrum für Europäische Sozialforschung 22, Mannheim 2000. 
Die Ursache für diese Abweichungen könnte jedoch in der wirtschaftlichen Lage in Ostdeutschland zu suchen sein. Da mehr Menschen von Arbeitslosigkeit und Armut bedroht sind, könnte dies die Zustimmung zum Sozialstaat erklären. In Tabelle 6 werden deshalb die Einstellungen zum Sozialstaat unter Einbezug sozioökonomischer Kontrollvariablen getestet. Die Spaltennummern beziehen sich dabei auf die in Tabelle 5 aufgeführten Fragen und gruppieren diese hinsichtlich des zugrunde liegenden Sozialstaatsverständnisses. ${ }^{45}$ Auch unter Berücksichtigung weiterer Faktoren unterscheiden sich die Einstellungen zwischen Ost- und Westdeutschen. Erstere weisen häufiger eine umfassende Sozialstaatsorientierung auf und stimmen mit geringerer Wahrscheinlichkeit Aussagen zu, die eine Reduzierung sozialer Leistungen beinhalten. Dasselbe gilt für Frauen. Sie sind durchweg stärker sozialstaatsorientiert als Männer. Wie oben bereits gezeigt, spiegeln sich diese Präferenzen im Wahlverhalten wider. Frauen wählen überproportional SPD und Grüne. Aus Tabelle 6 spricht zudem eine eigeninteressierte Bewertung des Sozialstaats. Mit höherer Bildung und höherem Einkommen steigt die Wahrscheinlichkeit, einen nur moderat ausgestalteten Sozialstaat zu unterstützen.

\begin{tabular}{|c|c|c|c|c|c|c|}
\hline \multicolumn{7}{|c|}{ Tabelle 6: Determinanten der Zustimmung zum Sozialstaat in der Bundesrepublik 2004} \\
\hline & \multicolumn{3}{|c|}{ umfassend } & \multicolumn{3}{|c|}{ gemäßigt } \\
\hline & (1) & (2) & (3) & (4) & (5) & (6) \\
\hline $\begin{array}{l}\text { Alter } \\
\text { (in Jahren) }\end{array}$ & $\begin{array}{c}1,002 \\
(0,003)\end{array}$ & $\begin{array}{l}0,986^{* * *} \\
(0,004)\end{array}$ & $\begin{array}{l}1,006^{*} \\
(0,003)\end{array}$ & $\begin{array}{l}1,013^{* * *} \\
(0,003)\end{array}$ & $\begin{array}{c}1,002 \\
(0,003)\end{array}$ & $\begin{array}{l}1,011^{* * *} \\
(0,003)\end{array}$ \\
\hline $\begin{array}{l}\text { Geschlecht } \\
\text { (1=weiblich) }\end{array}$ & $\begin{array}{l}1,269^{* *} \\
(0,083)\end{array}$ & $\begin{array}{l}1,546^{* * *} \\
(0,116)\end{array}$ & $\begin{array}{l}1,721^{* * *} \\
(0,091)\end{array}$ & $\begin{array}{l}0,708^{* * *} \\
(0,091)\end{array}$ & $\begin{array}{c}0,923 \\
(0,083)\end{array}$ & $\begin{array}{l}0,746^{* * *} \\
(0,084)\end{array}$ \\
\hline $\begin{array}{l}\text { Bildungsgrad } \\
\text { (1=niedrig; } 5=\text { hoch) }\end{array}$ & $\begin{array}{l}0,720^{* * *} \\
(0,042)\end{array}$ & $\begin{array}{l}0,747^{* * *} \\
(0,051)\end{array}$ & $\begin{array}{l}0,719^{* * *} \\
(0,042)\end{array}$ & $\begin{array}{l}1,247^{* * *} \\
(0,043)\end{array}$ & $\begin{array}{c}0,945 \\
(0,040)\end{array}$ & $\begin{array}{l}0,863^{* * *} \\
(0,040)\end{array}$ \\
\hline $\begin{array}{l}\text { Einkommen } \\
\text { (1=niedrig; } 5=\text { hoch })\end{array}$ & $\begin{array}{l}0,885^{* * *} \\
(0,029)\end{array}$ & $\begin{array}{l}0,794^{* * *} \\
(0,045)\end{array}$ & $\begin{array}{l}0,742^{* * *} \\
(0,034)\end{array}$ & $\begin{array}{c}1,049 \\
(0,033)\end{array}$ & $\begin{array}{r}1,075^{*} \\
(0,030)\end{array}$ & $\begin{array}{l}1,016 \\
(0,030)\end{array}$ \\
\hline $\begin{array}{l}\text { politische Orientierung } \\
(1=\text { links; } 10=\text { rechts })\end{array}$ & $\begin{array}{l}0,918^{* *} \\
(0,025)\end{array}$ & $\begin{array}{l}0,889^{* *} \\
(0,034)\end{array}$ & $\begin{array}{l}0,884^{* * *} \\
(0,027)\end{array}$ & $\begin{array}{l}1,147^{* * *} \\
(0,027)\end{array}$ & $\begin{array}{l}1,224^{* * *} \\
(0,025)\end{array}$ & $\begin{array}{l}1,172^{* * *} \\
(0,026)\end{array}$ \\
\hline $\begin{array}{l}\text { Region } \\
(1=\text { Ost })\end{array}$ & $\begin{array}{l}1,323^{* *} \\
(0,089)\end{array}$ & $\begin{array}{l}2,395^{* * *} \\
(0,146)\end{array}$ & $\begin{array}{l}1,814^{* * *} \\
(0,103)\end{array}$ & $\begin{array}{l}0,349^{* * *} \\
(0,110)\end{array}$ & $\begin{array}{l}0,477^{* * *} \\
(0,090)\end{array}$ & $\begin{array}{l}0,582^{* * *} \\
(0,089)\end{array}$ \\
\hline Nagelkerke $\mathrm{R}^{2}$ & 0,079 & 0,106 & 0,167 & 0,109 & 0,091 & 0,079 \\
\hline $\mathrm{N}$ & 2541 & 2576 & 2537 & 2535 & 2528 & 2511 \\
\hline \multicolumn{7}{|c|}{$\begin{array}{l}{ }^{*} \mathrm{p}<0,05 ;{ }^{* *} \mathrm{p}<0,01 ;{ }^{* * *} \mathrm{p}<0,001 \text {. } \\
\text { Angegeben wird, inwiefern die unabhängigen Variablen die Wahrscheinlichkeit verändern, dass eine } \\
\text { Person den in der Tabelle aufgeführten Aussagen zustimmt. Die Werte entsprechen unstandardisierten } \\
\text { Odds Ratios; in Klammern: Standardfehler. } \\
\text { Quelle: Eigene Berechnungen auf Basis der Daten vom ALLBUS 2004, ZA-Nr. } 3762 \text {. }\end{array}$} \\
\hline
\end{tabular}

45 Die Bündelung der Variablen in zwei Gruppen wurde durch eine explorative Faktorenanalyse vorgenommen. Um den Informationsverlust zu vermeiden, der mit der Reduktion auf zwei Faktoren einhergeht, wurden auch in Tabelle 6 alle sechs Variablen wiedergegeben. 
Angesichts dieser Einstellungen dürften Einschnitte in die Systeme sozialer Sicherung politisch schwierig durchzusetzen sein. Das Problem der Volksparteien ist, dass die „Finanzkrise des deutschen Staats “46 Kürzungen erfordert, die von ihren Wählern nicht gutgeheißen werden. Die Anhänger von Bündnis 90/Die Grünen und der FDP sind aufgrund ihres überdurchschnittlichen Einkommens seltener persönlich von Einschnitten betroffen und strafen ihre Partei für die Reform des Sozialstaats nicht ab. Die Linkspartei profitiert davon, Sprachrohr der Unzufriedenen mit der Sozial- und Arbeitsmarktpolitik zu sein. ${ }^{47}$ Im Ergebnis verzeichnen die kleinen Parteien hohe Zustimmungswerte in Umfragen, obwohl (oder weil) sie wenig Einfluss auf die politischen Entscheidungen ausüben können. Die Große Koalition im Bund stärkt zudem die kleinen Parteien und erhöht damit die Wahrscheinlichkeit ihrer eigenen Fortsetzung über 2009 hinaus, so lange Dreierbündnisse am Widerstand der kleinen Parteien scheitern.

\section{Konsequenzen für die Volksparteien}

Die deutschen Wähler sind in ihren Einstellungen zur Wirtschafts- und Gesellschaftspolitik gespalten. Eine Mehrheit unterstützt in wirtschafts- und sozialpolitischen Fragen eher linke, gesellschaftspolitisch jedoch konservative Positionen. Vor diesem Hintergrund weisen Franz Urban Pappi und Susumu Shikano darauf hin, dass die Wahlkampfstrategie der CDU fehlgeleitet war, mit einem wirtschaftsliberalen Reformprogramm eine Mehrheit zu suchen. ${ }^{48}$ Stattdessen hätte sie gesellschaftspolitische Themen hervorheben sollen. Aus dieser Analyse ergibt sich ein Konflikt für die Unionsparteien zwischen Stimmen- und Machtorientierung (vote- und office-seeking). Mit der Betonung gesellschaftspolitischer Themen haben sie bessere Chancen, ihren Stimmenanteil zu maximieren. Gleichzeitig verringert eine solche Strategie die Koalitionsoptionen, weil eine Zusammenarbeit mit den Grünen erschwert wird, die diesbezüglich am anderen Ende des politischen Spektrums stehen. Geht man davon aus, dass nach der nächsten Wahl eine ähnliche Sitzverteilung im Bundestag wie heute besteht, erscheint die Große Koalition als einzige Möglichkeit, Stimmen- und Machtorientierung in Einklang zu bringen. Die relative Schwäche der Union liegt in dieser Konstellation darin, dass diese Option nur realisiert werden kann, wenn die SPD keine Ampel- oder Linkskoalition eingeht.

Die vorgestellten Befunde lassen für die SPD eine Zusammenarbeit mit der Linkspartei als strategisch vorteilhaft erscheinen. In allen anderen Koalitionen ist sie zu Reformen gezwungen, die die eigene Kernklientel enttäuschen und die Beziehung zu den Gewerkschaften belasten. In einem Dreierbündnis mit den Grünen und der Linkspartei wäre letz-

46 Wolfgang Streeck, Endgame? The Fiscal Crisis of the German State, in: MPIfG Discussion Paper 07/7, Köln 2007.

47 Wo sie in Regierungsverantwortung selbst zu Sparprogrammen gezwungen war, verlor sie in der Wählergunst und sah sich linkem Protest zum Beispiel durch die WASG ausgesetzt. Bei der Berliner Abgeordnetenhauswahl 2006 verlor die Linkspartei im Ostteil der Stadt im Vergleich zu 2001 fast die Hälfte ihrer Wähler. Vgl. Oskar Niedermayer / Richard Stöss, Die Berliner Abgeordnetenhauswahl vom 17. September 2006: Ein „Weiter so" trotz herber Verluste des Koalitionspartners, in: ZParl, 38. Jg. (2007), H. 1, S. 84 ff.

48 Vgl. Franz Urban Pappi / Susumu Shikano, Regierungsabwahl ohne Regierungsneuwahl, in: PVS, 46. Jg. (2005), S. 513 - 526, S. 517. 
tere gezwungen, unpopuläre Beschlüsse mitzutragen. Jenseits dieser machtpolitischen Überlegungen ergibt sich aus der Sozialstaatsorientierung der Bevölkerung eine denkbare Reformagenda für ein Linksbündnis, die dem Wählerwillen näher stünde als die einer Ampel- oder „Jamaika“-Lösung. Eine Mitte-links-Koalition könnte den Sozialstaat in Richtung des universalistischen, sozialdemokratischen Modells umbauen. Elemente desselben wären unter anderem eine höhere Steuerfinanzierung der sozialen Sicherung, die Einführung einer Bürgerversicherung beispielsweise in den Feldern Gesundheit und Pflege, der Ausbau eines öffentlichen Beschäftigungssektors, umfassende Kinderbetreuung und Investitionen in das Bildungssystem. Die im vorherigen Abschnitt zitierten Meinungsumfragen legen nahe, dass diese Strategie auf eine wohlwollende Beurteilung der Bevölkerungsmehrheit stieße.

Die Reform des deutschen Sozialstaats ist für beide Volksparteien riskant. Streit über den richtigen Kurs herrscht nicht nur in der SPD, sondern auch in der CDU - die Heftigkeit ist bei den Sozialdemokraten größer, weil sie schlechte Umfragewerte erhalten und das Verhältnis zu den Gewerkschaften belastet ist. Beides sorgt innerhalb der Partei für Unruhe und führt zu öffentlichen Auseinandersetzungen. Aber auch in der CDU kann das Bekenntnis zur sozialen Marktwirtschaft nur mühsam die Spannung zwischen liberalen Reformern und dem christlich-sozialen Arbeitnehmerflügel überdecken. Weitere soziale Einschnitte fördern zudem die Abwanderung der Wähler zu den kleinen Parteien. Die Rückkehr zu einem Drei- oder Vierparteiensystem ist daher nicht zu erwarten. Um sich auf diese Situation einzustellen, werden sich beide Volksparteien neuen Koalitionsvarianten öffnen müssen. Für die CDU legt dies eine Annäherung an die Grünen nahe. Gelingt dies, stellt sich für die SPD umso dringlicher die Frage, ob sie auf Bundesebene den Abgrenzungskurs gegen die Linke beibehalten kann. 